\title{
COINFECCIÓN POR DENGUE Y LEPTOSPIROSIS EN UNA NIÑA DE LA AMAZONÍA PERUANA
}

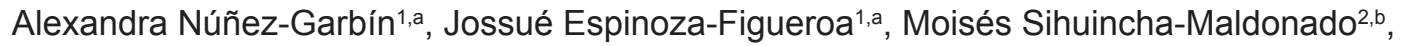 \\ Luis Suarez-Ognio ${ }^{1, c}$
}

\begin{abstract}
RESUMEN
Se reporta el caso de una niña de 10 años, natural y procedente de la ciudad de lquitos en Perú que presentó cefalea, fiebre, escalofríos, dolor osteomuscular, leve dolor en epigastrio, epistaxis y hematemesis. Al examen físico la paciente se encontraba afebril y en regular estado general. Por medio de pruebas serológicas se confirmó la infección por dengue y leptospirosis. La paciente recibió hidratación endovenosa con cloruro de sodio al 0,9\% y penicilina G sódica, logrando una evolución clínica favorable por lo que fue dada de alta a los pocos días de su ingreso al hospital. Aunque estas dos enfermedades son comunes en la Amazonía peruana, la presencia simultánea de ambas en la población pediátrica es poco documentada; por ello, una buena historia clínica y exámenes de laboratorio son importantes para el diagnóstico y tratamiento oportuno.
\end{abstract}

Palabras clave: Leptospirosis; Dengue; Coinfección; Pediatría (fuente: DeCS BIREME)

\section{COINFECTION OF DENGUE AND LEPTOSPIROSIS IN A GIRL FROM THE PERUVIAN AMAZON}

\begin{abstract}
We report the case of a 10 year old girl, born and raised in the city of Iquitos in Peru who presented with headache, fever, chills, musculoskeletal pain, mild epigastric pain, epistaxis and hematemesis. On physical examination, the patient was afebrile and in good general condition. Serological tests confirmed infection of dengue and leptospirosis. The patient received intravenous hydration with sodium chloride $0.9 \%$ and penicillin $\mathrm{G}$ sodium, achieving a favorable clinical course such that she was discharged a few days after admission to the hospital. Although these diseases are common in the Peruvian Amazon, the simultaneous presence of both in the pediatric population is little documented; therefore, a good clinical history and laboratory tests are important for diagnosis and treatment.
\end{abstract}

Key words: Leptospirosis; Dengue; Coinfection; Pediatrics (source: MeSH NLM)

\section{INTRODUCCIÓN}

El dengue y la leptospirosis constituyen dos importantes causas de síndrome febril hemorragíparo indiferenciado, principalmente en las regiones subtropicales y tropicales (1). La incidencia anual estimada de casos de leptospirosis varía de 0,1 a 1 por cada 100000 habitantes en climas templados y de 10 a 100 por 100000 habitantes en climas húmedos tropicales. La incidencia aumenta a más de 100 por 100000 habitantes en casos de brotes y grupos con alto riesgo de exposición (2). En tanto, el dengue constituye una enfermedad endémica en América; entre los años 2008 y 2012 se han notificado una media de 1,15 millones de casos de dengue, 32301 casos de dengue grave y 726 muertes como consecuencia de esta enfermedad ${ }^{(3)}$.

En el Perú, de acuerdo con cifras de la Dirección General de Epidemiología (DGE), se presentaron 18 677 casos de dengue en el año 2012 (58\% confirmados y $42 \%$ probables), siendo el virus del dengue serotipo 2 el predominante. Asimismo, las regiones con un mayor número de estos fueron Loreto, Ucayali y Cajamarca (4). En cuanto a leptospirosis, se reportaron 1583 casos desde el 1 de enero hasta el 24 de agosto del año 2013 y las regiones más afectadas por esta enfermedad fueron Loreto, San Martín y Ayacucho ${ }^{(5)}$.

\footnotetext{
Escuela de Medicina, Universidad Peruana de Ciencias Aplicadas. Lima, Perú.

Hospital de Apoyo Iquitos. Loreto, Perú.

Médico cirujano; ${ }^{\mathrm{b}}$ médico infectólogo; ${ }^{\mathrm{c}}$ médico epidemiólogo

Recibido: 22-09-14 Aprobado: 04-03-15
}

Citar como: Núñez-Garbín A, Espinoza-Figueroa J, Sihuincha-Maldonado M, Suarez-Ognio L. Coinfección por dengue y leptospirosis en una niña de la Amazonía peruana. Rev Peru Med Exp Salud Publica. 2015;32(1):179-82. 
La coinfección por dengue y leptospirosis es una condición poco frecuente en pacientes pediátricos, de tal manera que en la literatura médica se ha registrado solo un estudio en niños tailandeses ${ }^{(6)}$. Cabe resaltar que no se han reportado publicaciones anteriores en nuestro país, o es posible que en el pasado se hayan presentado casos, pero no han sido adecuadamente documentados.

El objetivo de este reporte de caso es describir el cuadro clínico, la evolución y tratamiento de la coinfección por dengue y Leptospira en una paciente del servicio de pediatría del Hospital de Apoyo de Iquitos.

\section{REPORTE DEL CASO}

Mujer de 10 años de edad, natural y procedente de una zona urbana de lquitos, Perú, sin antecedentes patológicos de importancia. La madre de la paciente manifestó que 5 días antes del ingreso inició con episodios de fiebre precedidos por escalofríos, cefalea, dolor osteomuscular, náuseas y vómitos en escasa cantidad, sin sangre y sin moco. Al día siguiente de la aparición de los síntomas se agrega dolor de leve intensidad en el epigastrio por lo que acude a una posta en donde recibe metamizol y le dan de alta. La fiebre persistió y el día del ingreso se agregó epistaxis y hematemesis; motivo por el cual acudió al Servicio de Emergencia de Pediatría del Hospital de Apoyo Iquitos, en donde se decidió su hospitalización.

Al examen físico se evidenciaron funciones vitales estables, a excepción de una frecuencia cardiaca de 115 latidos por minuto y 42 respiraciones por minuto. La paciente se encontraba con una piel fría, sudorosa, ligeramente pálida y con un dolor de leve intensidad a la palpación en epigastrio.

El hemograma solicitado al ingreso mostró trombocitopenia de 125000 plaquetas $/ \mathrm{mm}^{3}$; hematocrito de $38 \%$; recuento de leucocitos de $8800 / \mathrm{mL}$ con $58 \%$ de neutrófilos, $34 \%$ de linfocitos y $8 \%$ de eosinófilos. En el examen de orina se evidenciaron 18 a 24 leucocitos por campo, con abundantes células epiteliales y de 10-12 hematíes por campo. El examen de gota gruesa para malaria fue negativo. La radiografía de tórax mostró un infiltrado intersticial bilateral (Figura 1).

A las $24 \mathrm{~h}$ del ingreso presentó una disminución del hematocrito a $32 \%$ y de plaquetas a $96000 / \mathrm{mm}^{3}$. El resultado de una nueva toma de gota gruesa para malaria también fue negativo.

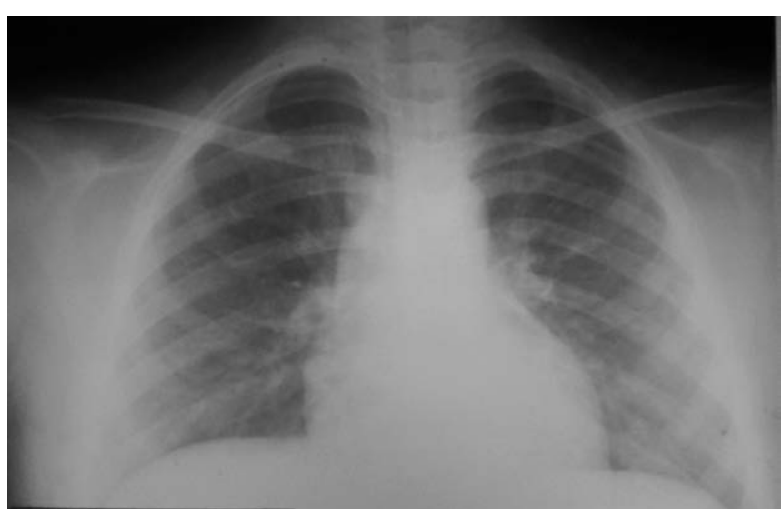

Figura 1. Radiografía de tórax de incidencia posteroanterior; muestra un infiltrado intersticial bilateral, a predominio de ambas bases pulmonares

A las $48 \mathrm{~h}$ el hematocrito nuevamente disminuyó a $28 \%$, las plaquetas se elevaron a $146000 / \mathrm{mm}^{3}$ y otros resultados mostraron: VSG $8 \mathrm{~mm} / \mathrm{h}$, urea $20 \mathrm{mg} / \mathrm{dL}$, creatinina $0,89 \mathrm{mg} / \mathrm{dL}$, proteínas totales $7,28 \mathrm{~g} / \mathrm{dL}$, albúmina 3,20 mg/dL, globulina 4,08 mg/dL, TGO $67 \mathrm{U} / \mathrm{L}$ y TGP 40 U/L. Un segundo examen de orina mostró 2-3 leucocitos por campo, células epiteliales escasas y no se observaron hematíes. La ecografía abdominal reveló una vesícula biliar con paredes edematizadas.

La paciente recibió hidratación endovenosa con solución salina al $0,9 \%$ y penicilina $G$ sódica 1,5 millones de unidades por vía intravenosa cada 6 h; mostró mejoría por lo que fue dada de alta a los 7 días del ingreso.

El laboratorio de referencia regional de Loreto confirmó la serología para Leptospira ELISA Ig $\mathrm{M}$ y dengue ELISA Ig $\mathrm{M}$.

\section{DISCUSIÓN}

Dentro de las causas de síndrome febril agudo en los trópicos, dengue y leptospirosis son dos enfermedades comunes y presentan características clínicas muy parecidas. Por tal motivo es difícil diferenciarlas en etapas tempranas ${ }^{(1,7)}$

Un estudio prospectivo en Mumbai describió un brote simultáneo de leptospirosis y dengue, el cual encontró que los hallazgos clínicos más asociados al primer cuadro fueron hemorragia, dolor abdominal, hepatoesplenomegalia, edema y sufusión conjuntival. En el caso del dengue, las condiciones más asociadas fueron trombocitopenia y presencia de artralgias. Finalmente, este estudio demostró que en los barrios bajos de la ciudad de Mumbai se produjo un brote simultáneo de leptospirosis y dengue ${ }^{(8)}$. 
Por otro lado, un estudio comparativo sobre dengue y Leptospira en niños de una región semirrural del centro-norte de Tailandia encontró que entre los niños que acuden a un hospital durante la temporada de monzones, la leptospirosis constituye al menos el $6 \%$ de todas las enfermedades febriles agudas indiferenciadas y el $19 \%$ de las enfermedades no dengue. Asimismo, la prevalencia de esta enfermedad en el entorno urbano de Bangkok fue menor que en el rural ${ }^{(6)}$.

Otro estudio realizado en Paraguay con 47 niños y adolescentes infectados por dengue, con una edad promedio de 10 años, encuentra que los síntomas más frecuentes fueron fiebre, cefalea, dolor retroocular, dolor osteomioarticular y exantema ${ }^{(9)}$. Cabe resaltar que los preescolares pueden mostrar un cuadro febril indiferenciado u oligosintomático ${ }^{(10)}$. Además, se reporta que la probabilidad de morir en niños es aproximadamente 15 veces mayor que en los adultos cuando padecen de una infección secundaria por dengue ${ }^{(11)}$.

Con respecto a la leptospirosis, esta es infradiagnosticada debido a que varios casos no presentan la forma ictérica severa ${ }^{(6)}$. Asimismo, se ha evidenciado que la enfermedad en niños es generalmente menos severa que en adultos y el sexo que predomina en aquellos que la padecen es el masculino ${ }^{(12)}$.

Cabe resaltar que uno de los síntomas principales que presentó la paciente fue fiebre, la cual es una manifestación común de diversas enfermedades infecciosas tropicales. En lugares rurales con escasos recursos para el diagnóstico, las etiologías son diversas e, incluso, algunas desconocidas. Dentro de las causas de fiebre indiferenciada en zonas tropicales se encuentran: malaria, rickettsiosis, leptospirosis, tifoidea, dengue y otras enfermedades virales. Dentro del grupo de enfermedad febril asociado a rash, trombocitopenia y disfunción multiorgánica, las etiologías más probables son malaria falciparum, leptospirosis y dengue, por lo que es necesario tenerlas en cuenta para un adecuado enfoque sindrómico ${ }^{(13)}$.

El diagnóstico de dengue y leptospirosis en nuestro reporte de caso se realizó mediante ELISA IgM. En el caso del primero, este puede realizarse en dos etapas de la enfermedad. En la etapa temprana, mediante la detección del ácido nucleico viral o del antígeno NS1; mientras que en la etapa final, el diagnóstico de elección es serológico. El uso de la prueba ELISA IgM en dengue presenta una sensibilidad de $21-99 \%$ y especificidad de $77-78 \%$. Dentro de sus limitaciones, se ha reportado la presencia de reacciones cruzadas en pacientes con malaria, leptospirosis e infección pasada por el mismo virus, por tal motivo este aspecto debe ser tomado en cuenta al realizarla en áreas donde circulan estos patógenos ${ }^{(14)}$. Por otro lado, el diagnóstico definitivo de leptospirosis se realiza a través del cultivo para Leptospira spp. o mediante la prueba de aglutinación microscópica. La Organización Mundial de la Salud (OMS) ha recomendado el diagnóstico serológico en zonas con recursos limitados, como en nuestro caso, donde el diagnóstico se realizó en el Laboratorio de Referencia Regional de Loreto. La prueba ELISA IgM, es la más utilizada para la detección de leptospirosis precoz, presenta una sensibilidad de $68,1 \%$ y una especificidad de $96,3 \%$ en etapas iniciales de la enfermedad ${ }^{(15)}$.

Se tiene poca información sobre la implicancia clínica de la coinfección por dengue y leptospirosis en niños. Esta última enfermedad puede presentarse de diferentes maneras que van desde pacientes asintomáticos, hasta formas graves como la enfermedad de Weill. Debe ser tratada simultáneamente con antibióticos, como en nuestro caso, donde se utilizó solución salina y penicilina $G$ sódica para una forma moderada o grave de la enfermedad. Los casos leves de leptospirosis en niños menores de 8 años pueden ser tratados con amoxicilina o azitromicina por vía oral y, en mayores, con doxiciclina por vía oral ${ }^{(13)}$.

Ambas enfermedades presentan manifestaciones clínicas similares durante la fase inicial, para lo cual los médicos deben de estar atentos, con el fin de evitar complicaciones. Asimismo, la presencia de lluvias favorece su transmisión lo cual aumenta la posibilidad que aparezcan coinfecciones (16). Es importante mencionar que las pruebas serológicas para ambas enfermedades deben realizarse de forma precoz para confirmar el diagnóstico.

Concluimos que, a pesar de ser estas dos entidades comunes en la Amazonía peruana, la coinfección por estos dos patógenos es poco documentada en niños; sin embargo, el médico debería tenerlas presente para ofrecer un tratamiento oportuno. Para esto, una buena historia clínica y exámenes de laboratorio son indispensables para su diagnóstico, los cuales deben de iniciarse ni bien se sospeche clínicamente ambas enfermedades en un paciente. La DGE implementó en el año 2005 la Vigilancia Sindrómica la cual incluye al síndrome febril, al síndrome febril ictérico y al síndrome febril hemorrágico, con el objetivo de sensibilizar la identificación temprana y respuesta inmediata de brotes por diferentes agentes etiológicos, incluidos el dengue y la leptospirosis. El cumplimiento de esta directiva permitirá un mejor conocimiento de la epidemiología de la coinfección por dengue y leptospirosis.

Conflictos de interés: los autores declaran no tener conflictos de interés.

Fuentes de financiamiento: autofinanciado. 


\section{REFERENCIAS BIBLIOGRÁFICAS}

1. Victoriano AF, Smythe LD, Gloriani-Barzaga N, Cavinta LL, Kasai $\mathrm{T}$, Limpakarnjanarat $\mathrm{K}$, et al. Leptospirosis in the Asia Pacific region. BMC Infect Dis. 2009 Sep 4;9:147. doi: 10.1186/1471-2334-9-147.

2. Dirección General de Epidemiología. Manual de Procedimientos Estandarizados para la Vigilancia Epidemiológica de la Leptospirosis [Internet]. México, D.F.: DGE; 2012 [citado el 23 de mayo de 2014]. Disponible en: http:// www.epidemiologia.salud.gob. $\mathrm{mx} / \mathrm{doctos} / \mathrm{infoepid/vig \_ epid}$ manuales/14_2012_Manual_ Leptospirosis_vFinal_21nov12.pdf

3. Organización Panamericana de la Salud. Situación de Dengue en Centroamérica y republica Dominicana [Internet]. Washington D.C.: OPS; 2013 [citado el 17 de mayo de 2014]. Disponible en: http://www.paho. org $/$ cor $/$ index.php?option $=$ com $\mathrm{d}$ o c m a n \& t a s k = d o c . download\&gid $=279 \&$ Itemid $=224$

4. Perú, Ministerio de Salud. Casos de dengue por departamentos Perú 2012 [Internet]. Lima: MINSA; 2012 [citado el 22 de mayo de 2014]. Disponible en: http://www.dge. gob.pe/vigilancia/sala/2012/SE41/ dengue.pdf

5. Dirección General de Epidemiología. Resumen de las enfermedades o eventos sujetos a vigilancia epidemiológica a la semana epidemiológica 34. Bol
Epidemiol (Lima). 2013;22(34):735-9.

6. Libraty DH, Myint KS, Murray CK, Gibbons RV, Mammen MP, Endy TP, et al. A comparative study of leptospirosis and dengue in Thai children. PLoS Negl Trop Dis. 2007 Dec 26; 1(3):e111.

7. Levett PN, Branch SL, Edwards CN. Detection of dengue infection in patients investigated for leptospirosis in Barbados. Am J Trop Med Hyg. 2000 Jan;62(1):112-4.

8. Karande S, Gandhi D, Kulkarni M, Bharadwaj R, Pol S, Thakare J, et al. Concurrent outbreak of leptospirosis and dengue in Mumbai, India, 2002. J Trop Pediatr. 2005 Jun;51(3):174-81.

9. Giménez V, Picagua E, Carpinelli M, Rovira C, Ferreira L. Hallazgos laboratoriales y referencia sintomatológica en población pediátrica durante la epidemia de dengue del año 2007. Pediatr (Asunción). 2007;38(1):12-6.

10. Barreto FR, Teixeira MG, Costa Mda C, Carvalho MS, Barreto ML. Spread pattern of the first dengue epidemic in the city of Salvador, Brazil. BMC Public Health. 2008 Feb 7;8:51. doi: 10.1186/1471-2458-8-51.

11. Iturrino-Monge $\mathrm{R}$, Avila-Agüero $\mathrm{ML}$, Avila-Agüero CR, Moya-Moya T, Cañas-Coto A, Camacho-Badilla K, et al. Seroprevalence of dengue virus antibodies in asymptomatic Costa Rican children, 2002-2003: a pilot study. Rev Panam Salud Publica. 2006 Jul;20(1):39-43.
12. Agésilas F, Gey F, Monbrunt A, Combes JC, Llanas B, Scholssmacher $\mathrm{P}$, et al. [Acute leptospirosis in children in Reunion Island: a retrospective review of 16 cases]. Arch Pediatr. 2005 Sep;12(9): 1344-8. [Artículo en Frances].

13. From: The Indian Society of Critical Care Medicine Tropical fever Group, Singhi S, Chaudhary D, Varghese GM, Bhalla A, Karthi N, et al. Tropical fevers: Management guidelines. Indian J Crit Care Med. 2014 Feb;18(2):62-9. doi: 10.4103/0972-5229.126074.

14. Hunsperger EA, Yoksan S, Buchy P, Nguyen VC, Sekaran SD, Enria DA, et al. Evaluation of commercially available anti-dengue virus immunoglobulin M tests. Emerg Infect Dis. 2009 Mar;15(3):436-40. doi: 10.3201/ eid1503.080923.

15. Perú, Ministerio de Salud. Norma técnica de salud para la atención integral de la persona afectada con leptospirosis humana. Lima: MINSA; 2006.

16. Kaur H, John M. Mixed infection due to leptospira and dengue. Indian J Gastroenterol. 2002 SepOct;21(5):206.

Correspondencia: Alexandra Gabriela Núñez Garbin

Dirección:

Teléfono: 954740423

Correo electrónico: alexandrang@gmail.com

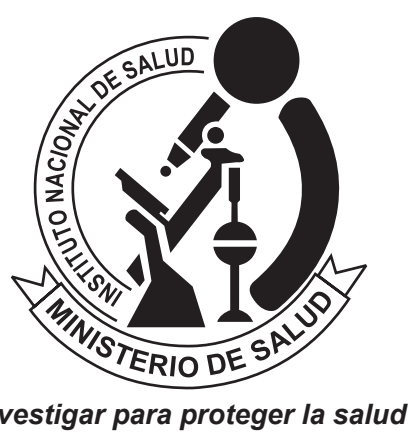

REVISTA PERUANA DE MEDICINA EXPERIMENTAL Y SALUD PÚBLICA CUMPLIENDO SUS METAS Y PROYECTÁNDOSE AL FUTURO

Visite los contenidos de la revista en: www.ins.gob.pe/rpmesp 